\title{
Una estructura neurodifusa para modelar la evapotranspiración instantánea en invernaderos
}

\section{A Neurofuzzy Structure Modelling Evapotranspiration in a Greenhouse Crop}

\author{
Ramos-Fernández J.C. \\ Universidad Politécnica de Pachuca, México. \\ E-mail:jramos@upp.edu.mx
}

López-Morales V.

Centro de Investigación en Tecnologías de Información y Sistemas-ICBI

Universidad Autónoma del Estado de Hidalgo, México.

E-mail:virgilio@uaeh.edu.mx

Lafont F.

Laboratoire des Sciences de L'Information et des Systémes UMR-CNRS 6168

Equipe COSI, Université de Sud-Toulon Var, La Garde.

E-mail:lafont@univ-tln.fr

Enea G.

Laboratoire des Sciences de L'Information et des Systémes UMR-CNRS 6168

Equipe COSI, Université de Sud-Toulon Var, La Garde.

E-mail:enea@univ-tln.fr

Duplaix J.

Laboratoire des Sciences de L'Information et des Systémes UMR-CNRS 6168

Equipe COSI, Université de Sud-Toulon Var, La Garde.

E-mail:duplaix@univ-tln.fr

(Recibido: septiembre de 2006; reevaluado: enero de 2009; aceptado octubre de 2009)

\section{Resumen}

En este artículo se reportan los resultados obtenidos de la experimentación con una planta de jitomate (Lycopersicon esculentum, Mill), en donde se propone una estructura neurodifusa para modelar la evapotranspiración instantánea. Se definen dos dinámicas de operación temporal (diurna y nocturna) en el modo de funcionamiento del sistema, se trabaja con un modelo de jerarquía difusa, así como una estructura de reglas Si-Entonces del tipo Takagi-Sugeno (T-S) con sus consecuentes lineales. La medición de la radiación solar se utiliza como selector difuso de las dos dinámicas de trabajo. La identificación de las premisas de las reglas difusas se obtiene mediante el algoritmo de clasificación difuso C-means y los parámetros de los consecuentes se determinan mediante el algoritmo de mínimos cuadrados ponderado por los valores de pertenencia. Se usa la variable del déficit de presión de vapor (DPV) para una mejor simplificación en la estructura neurodifusa del modelo de evapotranspiración. Esta variable se mide de manera indirecta usando las lecturas de la 
DOI: http://dx.doi.org/10.22201/fi.25940732e.2010.11n2.011

Una estructura neurodifusa para modelar la evapotranspiración instantánea en invernaderos

temperatura y la humedad relativa del invernadero, y así se obtiene una interpretación física del microclima que ayuda a preservar la calidad y sanidad del cultivo en el invernadero.

Descriptores: modelado jerárquico difuso, déficit de presión de vapor, evapotranspiración instantánea, algoritmo difuso de clasificación C-means.

\begin{abstract}
This paper shows the applicability of a neurofuzzy method applied to a tomato plant (Lycopersicon esculentum, Mill), in order to obtain a model of the instantaneous evapotranspiration. Two operational dynamics (diurnal and nocturnal) are defined in a hierarchical fuzzy model by a Takagi-Sugeno (T-S) type with linear consequents. The fuzzy selector of the two dynamics is the solar radiation measure. The fuzzy $C$-means algorithm is used to identify the fuzzy rules premises. The hierarchical fuzzy modelling is used to obtain a multi-model of the evapotranspiration cycles. In order to simplify the model structure, the variable of vapour pressure deficit (VPD) is introduced, and thus, a physical interpretation of the interior climate is obtained. VPD helps to preserve the quality and production level in the greenhouse crop.
\end{abstract}

Keywords: Hierarchical fuzzy modelling, vapour pressure deficit, instantaneous evapotranspiration, fuzzy C-means classification algorithm.

\section{Introducción}

El modelado de la evapotranspiración de cultivos en invernadero, ha sido estudiado por diversos autores con diferentes enfoques, así como el control del microclima, como se muestra en (Seginer, 2002), (Sigrimis et al., 2000), (Harmanto et al., 2004), (Körner et al., 2003), (Orgaz et al., 2004), (Jou et al., 2004), (Tantau et al., 2003), (Poss et al., 2004), (Abdulelah et al., 2004), (Medrano et al., 2004), (Roh et al., 1996). Algunos enfoques están orientados al uso eficiente de la energía para mantener la temperatura y la humedad (microclima) del invernadero en el rango deseable para una adecuada calidad en el cultivo, y de esta forma, disminuir la aplicación de agroquímicos para el control de plagas y enfermedades del cultivo. El microclima de un invernadero depende en parte de la dinámica de la evapotranspiración presente, la cual incluye: la transpiración de la planta y la evaporación del suelo, así como de las condiciones existentes en los mecanismos del invernadero (cortinas, domos, sombra, humidificadores y calefacción), también de las perturbaciones climáticas. Uno de los parámetros que mejor integran el estado de sanidad y desarrollo de un cultivo, es el DPV, el cual está ligado directamente con las dinámicas de evapotranspiración. En (Prenger et al., 2001) y (Körner et al., 2003) se muestra que el estado del cultivo se ve alterado por las variaciones extremas del microclima y la dinámica de evapotranspiración, condición que puede propiciar la incidencia de enfermedades y plagas. Un enfoque que ayuda a trabajar los ciclos de producción en invernadero de forma preventiva con resultados favorables, mediante el control del microclima y acciones fitosanitarias, se le conoce como manejo integrado de plagas (MIP) (Tantau et al., 2003). Asimismo, en (López et al., 2008) se considera un sistema inteligente para que a través de la AIP y el $D P V$, se realicen acciones preventivas en el cultivo de un invernadero. Una buena forma de prevenir enfermedades y plagas, así como mejorar la calidad y cantidad de la producción, es realizando el control en lazo cerrado del microclima con la retroalimentación del $D P V$. En (Körner et al., 2003) se reportan valores de referencia del $D P V$ entre 0.3-1.0 kPa que ayudan a mantener los cultivos sin problemas de enfermedad y con una adecuada transpiración de las plantas. En Sánchez y Rebolledo (2001) se reporta un rango de temperatura adecuado para el cultivo de jitomate en invernadero que debe estar en $16-24{ }^{\circ} \mathrm{C}$; sin embargo, las perturbaciones climáticas no lineales no siempre permiten mantener estos valores de temperatura en el rango de interés. Es por esto que en (Gary, 2001) se ilustra cómo la integración de la radiación solar es una variable importante en las estrategias para el control de riego. Recientemente, algunas técnicas basadas en inteligencia artificial (IA) y algoritmos computacionales han sido utilizadas con resultados alentadores en el modelado y control del 
DOI: http://dx.doi.org/10.22201/fi.25940732e.2010.11n2.011

Ramos-Fernández J.C., López-Morales V., Lafont F., Enea G. y Duplaix J.

microclima, la evapotranspiración y control de la irrigación en invernadero, como se ilustra en (Bahat et al., 2000), (Abdulelah et al., 2004), (Castañeda et al., 2006), (Trajkovic et al., 2000), (Salgado et al., 2004), (Jou et al., 2004). Por ejemplo, en Salgado et al. (2004) se propone un modelo con el enfoque de jerarquía difusa para aproximar la temperatura de un invernadero, en donde la dinámica del modelo de la temperatura se separa en dos regímenes de trabajo: temperatura con ventilación forzada y temperatura sin ventilación forzada. En Roh et al. (1996) utilizan la integración de la radiación solar para controlar la frecuencia en los ciclos de riego en un cultivo de pepinos. En Sigrimis et al. (2000) utilizan la integración de la temperatura en periodos de días (1, 2, 3, o más), para realizar el control de la temperatura con un enfoque de ahorro en el consumo de energía. En Gary (2001) se reportan algunas estrategias para realizar el control de los ciclos de riego y la nutrición en donde se citan ejemplos para la producción de jitomate en invernadero.

En el presente trabajo se realiza la integración de la radiación solar entre cada ciclo de riego, la cual se usa como una variable de entrada al modelo que se propone, donde se ilustra que la radiación solar es la variable que más peso tiene en las estrategias para el control del riego, mientras que los sistemas de control en lazo abierto mediante temporizadores (de uso bastante extendido actualmente) no responden adecuadamente a las necesidades de riego del cultivo. En Flores et al. (2005) se efectúa la integración de la radiación solar como un parámetro para realizar el control de los ciclos de riego a una producción de jitomate en invernadero. Este trabajo presenta también la integración de la radiación entre cada ciclo de riego, que se utiliza como una variable de entrada al modelo neurodifuso de evapotranspiración instantánea. Otro enfoque para programar los ciclos de irrigación, se presenta en Abdulelah et al. (2004), en donde se propone un modelo difuso para usar el índice de estrés hídrico foliar del cultivo (por sus siglas al inglés CWSI) como indicador del momento del riego, donde se mide la temperatura foliar del cultivo con un termómetro infrarrojo, entre otras diferentes variables del microclima. El CWSI toma valores desde cero hasta uno, el valor ideal se considera cercano a cero (sin estrés hídrico), cuando el valor del CWSI es uno es letal para el cultivo. Sin embargo, en Abdulelah et al. (2004) no se indica una metodología para determinar las necesidades hídricas de la planta y solamente proponen que el parámetro CWSI sea un valor cercano a cero para mantener sin estrés hídrico el cultivo. Así, proponen un modelo tipo Mamdani con 150 reglas difusas, con lo cual es claro que no es una tarea simple la programación de un microcontrolador comercial.

El trabajo que se reporta en este artículo propone una estructura neurodifusa para modelar la evapotranspiración de forma instantánea de una planta, que utiliza el $D P V$ como una entrada al modelo neurodifuso, el cual puede ser tomado como la referencia para un cultivo de la misma variedad bajo invernadero. Se utilizan las mediciones del microclima de un invernadero experimental tipo Richel de La Université du Sud-Toulon Var-Francia, así como registros de una balanza electrónica de alta precisión ( $\pm 0.325 \mathrm{gr}$ a $100 \mathrm{~kg}$ ) para medir la masa de la planta. Esta es una forma de medir la evapotranspiración de manera directa, también se le conoce como método lisimétrico (Coras, 2000).

\section{Cálculo del déficit de presión de vapor}

En la estructura del modelo neurodifuso que aquí se propone, es de especial interés medir en tiempo real el déficit de presión de vapor ( $D P V)$, y para medirlo de forma indirecta, se utilizan las mediciones del invernadero de: la temperatura en grados Celsius $\left({ }^{\circ} \mathrm{C}\right)\left(T_{i}\right)$ y la hu$\operatorname{medad}\left(H R \%=H_{i}\right)$ del invernadero. Se puede lograr una mejor interpretación de la dinámica evapotranspirativa de la planta, si se mide la temperatura foliar con instrumentos del tipo infrarrojo o láser.

Así, en (Prenger et al., 2001) se calcula el DPV mediante una tabla psicrométrica y mediante las ecuaciones (1)-(4).

$$
\begin{aligned}
& T=1.8 \cdot T_{i}+491.67 \\
& e_{s}=6.8947 \cdot e^{\left(A / T+B+C T+D T^{2}+E T^{3}+F \ln (T)\right)} \\
& e_{a}=e_{s} \cdot H_{i} / 100 \\
& D P V=6.894 \cdot\left(e_{s}-e_{a}\right)
\end{aligned}
$$

donde $T$ es la temperatura en grados Rankine, $e_{s}$ es la presión de vapor saturado y $e_{a}$ es la presión de vapor del aire ambas en $k P a$ y las constantes $A=-1.040 \times 10^{4}$, $B=-11.294, C=-0.0270, D=-1.289 \times 10^{-5}, E-2.478 \times 10^{-9}$, $F=-6.545$ y $D P V(k P a)$.

Un parámetro básico en el cálculo de la evapotranspiración es el índice de área foliar (IAF), el cual es una relación del área foliar por metro cuadrado de tierra. Es conveniente incluir el IAF en la estructura del modelo de evapotranspiración y microclima de un invernadero, ya que éste representa la densidad del cultivo, si se desea 
obtener un modelo de evapotranspiración de un ciclo completo de producción. En la estructura de modelado neurodifuso de evapotranspiración instantánea que se reporta en este trabajo, no se considera el IAF, puesto que la variación en el tamaño de la planta no es considerable entre cada ciclo de irrigación.

\section{Materiales y métodos}

Se instaló una maceta con una planta de jitomate variedad corazón de toro y substrato inerte (perlita, tipo B 0-1.5 mm, 80-90 kg/ $\mathrm{m}^{2}$ ) sobre una balanza electrónica dentro del invernadero, durante el mes de junio de 2005. Se realizaron mediciones de la masa cada minuto. Con un sistema de computadoras personales (PC) conectadas a una red de comunicación interna (Intranet) y un sistema de adquisición de datos analógicos y digitales, se midieron las variables del microclima y se ejecutaron las acciones de control del microclima invernadero. La gestión para la adquisición de datos y el control de los ciclos de irrigación (en lazo abierto) se desarrollaron con el software LabVIEW Ver. 6.1. El hardware de adquisición de datos de las variables climáticas se realizó con el sistema modular FP-2000 de National Instruments. Los sensores para medir las variables ambientales y el microclima del invernadero son del tipo analógico, los cuales envían la información eléctrica normalizada dentro del rango 4-20 mA hacia el sistema de adquisición de datos.
En una PC se guardaron las mediciones de la masa, el microclima, las variables ambientales y las acciones de control del sistema de sombra y la ventilación cenital. Naturalmente, la primera variable calculada fue el $D P V$ para las condiciones existentes en el invernadero.

En la figura 1 se muestran algunas mediciones de la humedad relativa $\left(H_{i}\right)$, la temperatura $\left(T_{i}\right)$ del invernadero y el $D P V$ calculado con (1)-(4).

Se observa que la $\left(H_{i}\right)$ tiene valores inferiores de $20 \%$, al mismo tiempo la $T_{i}$ alcanza $n$ valores por arriba de $45^{\circ} \mathrm{C}$. Así, el $D P V$ adquiere valores máximos de alrededor de $8.0 \mathrm{kPa}$ durante el periodo de altas temperaturas. Estos valores de temperatura y humedad son extremos para un cultivo agrícola en invernadero.

Observe que a medida que la temperatura incrementa la humedad disminuye. Estos valores tomados como referencia no son comunes en un invernadero manejado correctamente y con sistemas de regulación en lazo cerrado.

Enseguida y mediante un algoritmo en computadora, se realizó la aplicación de riego con un control clásico encendido-apagado, que se programó para activarse cada hora. La solución que se utilizó para irrigar la planta se preparó para la nutrición de jitomate, en formulación similar a la que se indica en (Sánchez y Rebolledo, 2001). Así mismo, se cuidó que el potencial de hidrogeno $(\mathrm{pH})$ del agua estuviera dentro del rango sugerido para el cultivo de jitomate.
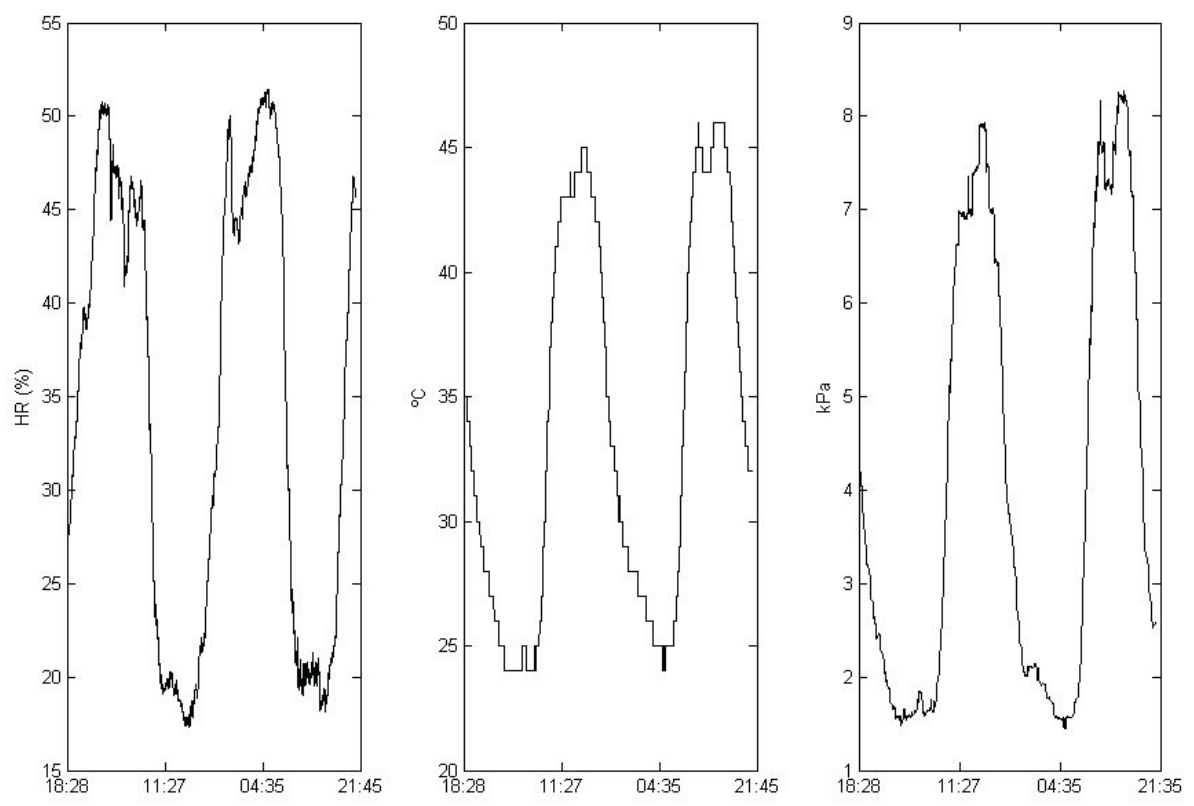

Figura 1. Humedad relativa $(\mathrm{Hi} \%)$, temperatura $\left(\mathrm{Ti}{ }^{\circ} \mathrm{C}\right)$ y $\mathrm{DPV}(\mathrm{kPa})$. 
En la figura 2 se muestra el sistema que se utilizó para realizar la experimentación, en donde se observa la balanza electrónica, la maceta con la planta, la guía tutor de la planta y el depósito del agua drenada. A la izquierda, se encuentra el depósito de agua con la solución nutrimental y una pequeña bomba eléctrica.

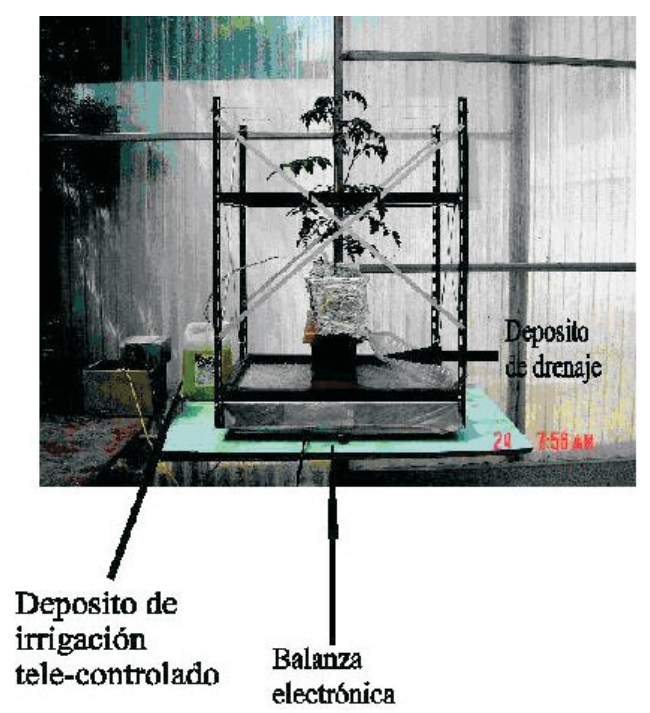

Figura 2. Planta de jitomate, sistema de riego y balanza electrónica

El agua drenada se conservó sobre la balanza electrónica en un depósito como se ve en la figura 2 , ya que de esta forma se puede cuantificar el total de agua drenada al final de los ciclos de riego. También se realizaron ciclos de riego con cantidades de agua conservadoras para evitar el drenado.

Como se recomienda en Nuez et al. (2001), el volumen de agua disponible para el experimento se inició con sustrato saturado de agua, y durante toda la experimentación se caracterizó la evapotranspiración del conjunto sustrato-planta-microclima. De esta forma, se probaron dinámicas de evapotranspiración desde condiciones con saturación de agua en el sustrato hasta condiciones de estrés de la planta, que permiten así verificar la robustez de la estructura del modelo que se propone en este trabajo.

Lo anterior, obedece a que en las técnicas de modelado neurodifuso con aprendizaje mediante mediciones del proceso, se sugiere excitar al sistema en los modos de operación que se desean modelar e identificar para asegurar que las dinámicas que se aproximan con la estructura del modelado sean muy apegadas a las dinámicas reales (Babuška, 1998).

\section{Desarrollo de la instrumentación virtual para la irrigación}

Se realizaron diferentes manipulaciones con el sistema de irrigación para caracterizar el caudal entregado por una pequeña bomba eléctrica alimentada con un voltaje de 5 volts y que es controlada (encendido/apagado) con una tarjeta de adquisición de datos que fabrica la empresa Rabit ${ }^{\circledR}$, que cuenta con un puerto de comunicación TCP conectada a una PC remota. Mediante interpolación lineal y utilizando diferentes mediciones de la masa agregada en gramos $(g r)$ de agua y el tiempo de encendido de la pequeña bomba en segundos (s), se caracterizó el caudal entregado por la bomba eléctrica de acuerdo a (5).

$$
Q(t)\left\{\begin{array}{l}
4.137 t-1.625 ; \text { si bomba }=1 \\
0 ; \text { si bomba }=0
\end{array}\right.
$$

donde $Q(t)$ es el caudal entregado por la bomba medido en gramos por segundo $(g r / s), t$ es el tiempo que dura encendida la bomba en segundos. La simulación del modelo de aproximación del caudal entregado por la pequeña bomba y los datos reales, se muestran en la figura 3.

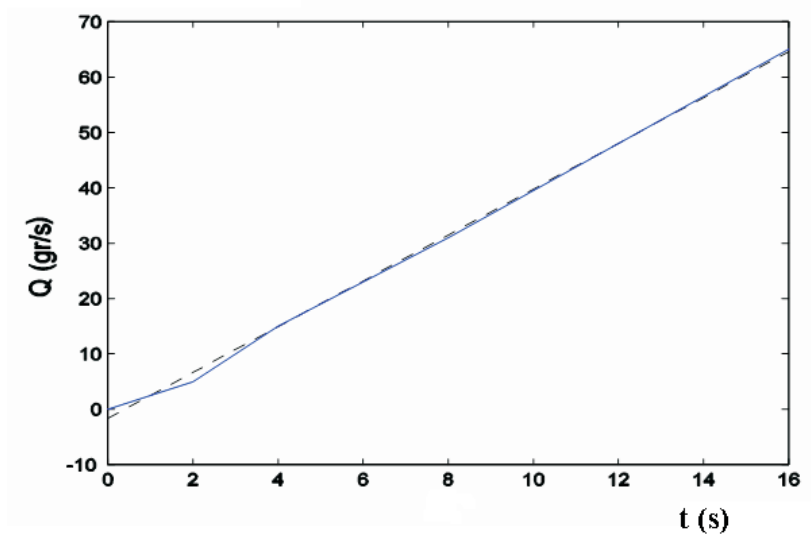

Figura 3. Linea continúa caudal real, línea punteada caudal simulado

En un sistema de producción con un invernadero comercial equipado con sistema de riego por goteo, se debe realizar la caracterización del caudal de los goteros, considerando que son autocompensantes. De esta forma, se puede obtener una ecuación que represente al caudal de agua adicionado a cada planta en función del tiempo de encendido-apagado del motor-bomba del sistema de irrigación. Utilizando (5) se desarrolló un instrumento virtual en ambiente de programación de 
LabVIEW, para efectuar los ciclos de irrigación, y donde se pueden reprogramar los ciclos de irrigación (en segundos), que se realizan cada hora, a partir de las 6:00 hasta las 22:00 horas.

\section{Estructura neurodifusa de modelado utilizando mediciones del sistema}

Tradicionalmente, el arte de modelar sistemas ha sido tratado como un enlace del conocimiento con herramientas matemáticas y la observación en el comportamiento del sistema a modelar. El modelo neurodifuso tipo T-S de evapotranspiración instantánea que se propone en este artículo describe las relaciones entre las variables del sistema por medio de reglas difusas Si-Entonces, como se ilustra a continuación:

$$
R_{i}: S_{i} X \text { es } A_{i} \text { Entonces } Y_{i}=X \theta_{i}
$$

donde $R_{i}$ es la regla que describe el i-ésimo submodelo, $X$ es un vector donde se encuentran todas las variables medidas, $A_{i}$ es la categoría similar o característica común del conjunto de variables $X, Y_{i}$ es la variable de salida del sistema y $\theta_{i}$ es el vector de parámetros del submodelo de aproximación.

Una ventaja de la estructura de modelado que aquí se propone sobre otras metodologías donde se utilizan parámetros que se describen de forma empírica, v. gr. (Coras, 2001), es que con este modelo se puede realizar la aproximación de manera instantánea de la evapotranspiración.

La aproximación en tiempo real que se realiza para una planta puede extrapolarse para un gran número de plantas y obtenerse así, una buena aproximación de referencia en un cultivo dentro de un invernadero de dimensiones comerciales.

\section{Algoritmo de clasificación de dinámicas C-means difuso}

En (6) se observa que $A_{i}$ es la i-ésima clasificación de la premisa en la regla $R_{i}$, de las variables que son medidas para determinar $A_{i}$ se realizó un proceso de identificación. Para ello, se programó el algoritmo de clasificación C-means difuso que a continuación se describe.

Dada una matriz de mediciones $Z$ de un experimento real, que se puede escribir en forma matricial (7), donde las columnas son las muestras en el tiempo, mientras que las filas son las variables $Z \in \mathfrak{R}^{N \times n}$, donde $n$ es el número de variables y $N$ es el número de muestras.

$$
Z=\left[\begin{array}{cccc}
z_{11} & z_{12} & \ldots & z_{1 N} \\
z_{21} & z_{22} & \ldots & z_{2 N} \\
\vdots & \vdots & \cdots & \vdots \\
z_{n 1} & z_{n 2} & \cdots & z_{n N}
\end{array}\right]
$$

El objetivo de utilizar el algoritmo C-means difuso es hacer la clasificación en cúmulos de datos con similitud entre sí, donde este término se entiende como una similitud matemática que se calcula por medio de una norma métrica, y para el algoritmo C-means difuso, se utiliza la norma Euclidiana (Babuška, 1998), (Yen et al, 1999). Enseguida, se define el número de cúmulos o particiones difusas $1<c<N$, donde $c$ es el número de cúmulos en la estructura de reglas difusas T-S, donde cada cúmulo identifica la premisa de una regla. Se propone el exponente difuso $m>1$. Como C-means es un algoritmo iterativo, se fija una tolerancia $(\varepsilon)$ y un máximo número de iteraciones (l) para finalizar la búsqueda de los cúmulos con similitud entre sí. Se inicializa la matriz de partición difusa $U^{0}=\mu_{i k}^{0}$ con números aleatorios $\in[0,1]$.

Con el algoritmo C-means difuso se estiman los antecedentes de la estructura de reglas Si-Entonces, que son los centros de los cúmulos o prototipos de cúmulos de datos, como se describe en el siguiente algoritmo.

\section{Algoritmo C-means difuso}

Repetir para $l=1,2, \ldots$

Paso 1: Cálculo de los prototipos de los centros de cúmulos de datos

$v_{i}^{l}=\frac{\sum_{k=1}^{N}\left(\mu_{i k}^{l-1}\right)^{m} z_{k}}{\sum_{k=1}^{N}\left(\mu_{i k}^{l-1}\right)^{m}} ; 1 \leq i \leq c$.

Paso 2: Cálculo de las distancias

$D_{i k A}^{2}=\left(z_{k}-v_{i}^{l}\right)^{T} A\left(z_{k}-v_{i}^{l}\right) ; 1 \leq i \leq c, 1 \leq k \leq N$,

donde $A$ es la matriz identidad.

Paso 3: Cálculo de la matriz de partición difusa

$$
\begin{aligned}
& \text { Si } D_{i k A}>0 \text { para } 1 \leq i \leq c, 1 \leq k \leq N \\
& \mu_{i k}^{\prime}=\frac{1}{\sum_{j=1}^{c}\left(\frac{D_{i k A}}{D_{j k A}}\right)^{2 /(m-1)}} .
\end{aligned}
$$


DOI: http://dx.doi.org/10.22201/fi.25940732e.2010.11n2.011

Ramos-Fernández J.C., López-Morales V., Lafont F., Enea G. y Duplaix J.

De otra manera:

$$
\begin{aligned}
& \mu_{i k}^{\prime}=0 \text { si } D_{i k A}>0, y \mu_{i k}^{\prime} \in[0,1] \operatorname{con} \sum_{i=1}^{c} \mu_{i k}^{l}=1 \\
& \text { Hasta }\left\|U^{l}-U^{l-1}\right\|<\varepsilon .
\end{aligned}
$$

$$
\text { Fin del Algoritmo }
$$

El algoritmo $C$-means difuso converge hasta que se cumple la condición $\left\|U^{l}-U^{l-1}\right\|<\varepsilon$ ó bien hasta un número máximo de iteraciones $(l)$.

\section{Aplicación del algoritmo C-means difuso a las dinámicas del sistema}

En las pruebas realizadas a la estructura que se propone en este trabajo, el número máximo de iteraciones se prefijó en 50 iteraciones $(l=50)$ y el resultado en la convergencia del algoritmo no fue mayor a 30 iteraciones. Se observó que el tiempo de convergencia en la búsqueda de los prototipos de cúmulos fue menor al periodo de muestreo que se programó en el sistema de adquisición de datos para los experimentos efectuados. También, en las simulaciones que se realizaron con los datos de los experimentos, se concluyó que era suficiente hacer el aprendizaje con los datos de un ciclo de irrigación, que permiten hacer una buena aproximación del ciclo de irrigación futuro, lo cual hace factible la implementación del algoritmo en tiempo real. Aún así, en este trabajo se tomaron una gran cantidad de mediciones (3000), para que el aprendizaje de los submodelos se realizara durante siete ciclos de irrigación para el periodo diurno y 200 mediciones (3 horas con 20 minutos) para el periodo nocturno.

Así, se puede apreciar la robustez en la respuesta de la estructura de modelado neurodifusa de evapotranspiración instantánea que se propone, cuando se trabaja con un rango de datos mayor a la que se tendría con el algoritmo trabajando en tiempo real.

En la fase de validación con los parámetros determinados en el aprendizaje, el modelo neurodifuso de evapotranspiración instantánea, aproxima la evapotranspiración por más de un día y una noche. Es decir, se puede realizar el aprendizaje entre cada ciclo de riego y obtener un modelo que prediga la evapotranspiración antes del siguiente ciclo de riego. De esta forma, se puede conservar un déficit de agua cercana a cero, considerando la disponibilidad de agua en la maceta como se sugiere en Nuez et al. (2001). Aún así, es siempre recomendable tener un porcentaje de drenaje un poco mayor a cero (Sánchez y Rebolledo, 2001).

\section{Cálculo de los consecuentes lineales para el modelo difuso T-S}

La estimación de los parámetros consecuentes se realiza con el método de mínimos cuadrados ordinal, en cada cúmulo de datos identificado con el algoritmo C-means difuso se hace un mapeo con un submodelo lineal y en consecuencia le corresponde una regla Si-Entonces. Los vectores de mediciones del experimento a modelar son agrupados como se indica en (8) y (9)

$$
\begin{aligned}
& X=\left[\begin{array}{cccc}
x_{1}^{1} & x_{1}^{2} & \cdots & x_{1}^{n} \\
x_{2}^{1} & x_{2}^{2} & \cdots & x_{2}^{n} \\
\vdots & \vdots & \vdots & x_{2}^{n} \\
x_{N}^{1} & x_{N}^{2} & \cdots & x_{N}^{n}
\end{array}\right] \\
& Y=\left[\begin{array}{c}
y_{1} \\
y_{2} \\
\vdots \\
y_{N}
\end{array}\right],
\end{aligned}
$$

donde $X \in \mathfrak{R}^{N \times n}, N$ es el número de muestras tomadas del experimento para realizar el aprendizaje, es el número de vectores de las mediciones que se utilizan como entradas del modelo y $Y \in \mathfrak{R}^{N \times 1}$ es el vector de mediciones de la variable que se desea aproximar. Se utilizan los valores del grado de pertenencia en cada cúmulo de datos que se expresan en una matriz diagonal como en (10).

$$
W_{i}\left[\begin{array}{cccc}
\mu_{i 1} & 0 & \cdots & 0 \\
0 & & \cdots & 0 \\
\vdots & \vdots & \ddots & \vdots \\
0 & 0 & \cdots & \mu_{i N}
\end{array}\right],
$$

donde $\mu_{i k}$ son los valores de pertenencia calculados con el algoritmo de $C$-means difuso de la l-ésima iteración, $i=1,2, . ., c, k=1,2, \ldots, N$ es el índice de muestreo.

Finalmente, los parámetros consecuentes de cada submodelo, se calculan con el algoritmo de mínimos cuadrados (11).

$$
\theta_{i}=\left[X^{T} W_{i} X\right]^{-1} X^{T} W_{i} Y
$$

donde $\theta_{i} \in \mathfrak{R}^{n \times 1}$ es el vector de parámetros lineales que caracteriza al $i$-ésimo submodelo neurodifuso.

Es decir, un submodelo lineal corresponde a cada cúmulo de datos similares entre sí, representados por la 
estructura de reglas difusas T-S, como se muestra en (6). Así, particularmente en este caso, se tiene que cada regla tiene la siguiente estructura:

$$
R_{i}: \text { Si Xes } V_{i} \text { Entonces } Y_{i}=X \theta_{i} \text {, }
$$

donde $R_{i}$ es la regla que describe el $i$-ésimo submodelo. La premisa de la regla es: Si $X$ es $V_{i}$ donde $V_{i}$ es el vector de centros del $i$-ésimo cúmulo de datos. El consecuente de la $i$-ésima regla es Entonces $Y_{i}=X \theta_{i}$, donde $Y_{i}$ es la aproximación con la activación de la $i$-ésima regla, $X$ son las mediciones del sistema que son la entrada al modelo neurodifuso y $\theta_{i}$ es el vector de parámetros estimados mediante el algoritmo de mínimos cuadrados (11).

\section{Modelo de evapotranspiración instantánea}

Para obtener la estructura del modelo jerárquico neurodifuso de evapotranspiración instantánea del tipo T-S (12), se analizaron diferentes arquitecturas de entrada-salida con las variables medidas climáticas, las acciones de control del invernadero y la masa del conjunto maceta-planta, utilizando todas las variables disponibles del invernadero experimental. Se utilizó un primer conjunto de datos para hacer el aprendizaje y estimar los parámetros de las reglas difusas T-S. Con otro conjunto de datos se realizó la validación de los submodelos obtenidos. Para las arquitecturas que se proponen en este trabajo, se consideró el menor número de variables de entrada al modelo. La razón es que hay dos dinámicas naturales de evapotranspiración instantánea, una para el día (luz solar) y otra para la noche. Por la noche, la radiación solar es cercana a cero y normalmente no se realizan ciclos de irrigación.

En la figura 4 se muestra la arquitectura propuesta para el submodelo de evapotranspiración que corresponde a la dinámica diurna, donde $T_{i}(k)$ y $H_{i}(k)$ son la temperatura y la humedad relativa del interior del invernadero en el evento de tiempo $(k)$, respectivamente.

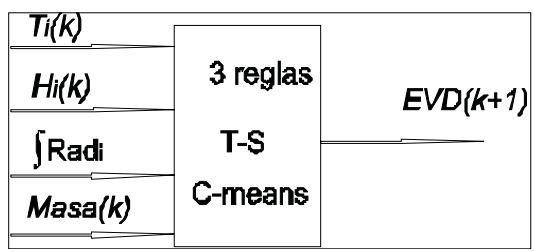

Figura 4. Submodelo de evapotranspiración para la dinámica del día
La variable Masa(k) es la masa de la maceta con la planta, $(\mathrm{kg})$ y $\int R a d_{i}$ es la integración de la radiación solar en el interior del invernadero $\left(\operatorname{Rad}_{i}(k)\right.$ en $\left.\mathrm{kW} / \mathrm{m}^{2}\right)$ que se calcula por medio de (13).

$$
\int R a d_{i}=\left\{\begin{array}{l}
0 ; \text { sibomba }=1 \\
k=K_{\text {boub } b=1} \\
\sum_{k=k_{0}} R a d_{i}(k) ; \text { si bomba }=0
\end{array},\right.
$$

donde $k_{0}$ es el primer instante después de la transición enciende (bomba =1) hacia apaga (bomba $=0)$ y $K_{\text {bomba }=1}$ es el instante en el cual se inicia el ciclo de irrigación. En los experimentos realizados se fijó un tiempo corto para esta transición $(\leq 9 s)$. El periodo de muestreo que se utilizó para la adquisición de datos fue de un minuto. Cada vez que hay un ciclo de irrigación se inicializa $\int R_{a} d_{i}=0$, es decir, un instante después cuando se adicionó agua a la planta se inicia la integración de la radiación solar hasta el siguiente ciclo de irrigación. Hay que remarcar que los resultados obtenidos utilizando la $\int$ Rad $_{i}$ como una entrada al modelo, mejoraron la aproximación del mismo con relación a las diferentes arquitecturas entradas-salida que se probaron con las mediciones disponibles del invernadero. Así, el submodelo para la dinámica diurna está en función de las cuatro variables, como se ilustra en (14).

$$
M D=\left[T_{i}(k) H_{i}(k) \int \operatorname{Rad}_{i} \operatorname{Mas} a(k)\right]
$$

En consecuencia, a través de este primer modelo se puede calcular la masa de agua evapotranspirada durante el día y durante el periodo de tiempo comprendido entre cada ciclo de irrigación por medio de (15).

$$
E V D(k+1)=\frac{\sum_{i=1}^{3} \mu_{i}^{d}(k)[M D] \theta_{i}}{\sum_{i}^{3} \mu_{i}^{d}}
$$

donde $E V D(k+1)$ es la evapotranspiración aproximada, $(\mathrm{kg}), \mu_{i}^{d}(k)$, es el valor de pertenencia del $i$-ésimo cúmulo del algoritmo C-means difuso, y

$\theta_{i}=\left[a_{i}^{T i} a_{i}^{H i} a_{i}^{\int R a d i} a_{i}^{\text {Masa }}\right]^{T}$

es el $i$-ésimo vector de parámetros de los consecuentes lineales obtenidos con aprendizaje local, con el método de mínimos cuadrados.

El submodelo para la dinámica nocturna, no incluye la variable $\int R a d_{i}$, ya que por la noche la radiación solar es casi nula. Así, la arquitectura del submodelo propuesto para la noche se muestra en la figura 5 . 


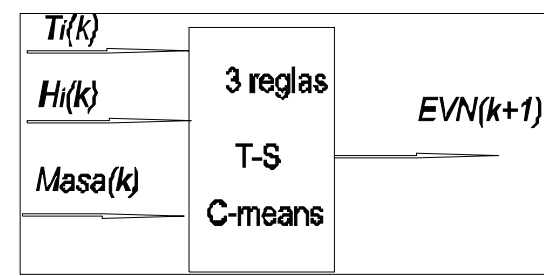

Figura 5. Submodelo de evapotranspiración para el periodo nocturno

La planta transpira cuando se encuentra hidratada de forma adecuada y cuando la planta tiene necesidad de agua y comienza a sufrir estrés hídrico, ésta se auto-protege hasta cierto punto de su deshidratación cerrando sus estomas por donde transpira y así auto-regula su temperatura biológicamente. El submodelo final para la dinámica nocturna está en función de tres variables, como se muestra en (16).

$$
M N=\left[T_{i}(k) H_{i}(k) \operatorname{Mas} a(k)\right]
$$

en consecuencia, a través del modelo obtenido se puede calcular la masa de agua evapotranspirada en la dinámica de noche y entre cada periodo de muestreo por medio de (17).

$$
\operatorname{EVN}(k+1)=\frac{\sum_{i=1}^{3} \mu_{i}^{n}(k)[M N] \beta_{i}}{\sum_{i=1}^{3} \mu_{i}^{n}(k)},
$$

donde $E V N(k+1)$ es la evapotranspiración en la noche (kg). El vector $\beta_{i}=\left[b_{i}^{T i} b_{i}^{\text {Hi }} b_{i}^{\text {Masa }}\right]^{T}$ son los parámetros de los consecuentes lineales T-S, de la $i$-ésima regla, con $i=1,2,3$ reglas.

El modelo global instantáneo se obtiene con la agregación jerárquica difusa de los submodelos con (18), donde se utiliza el valor de pertenencia de la radiación solar, que es la variable del selector difuso.

$$
\begin{aligned}
& E V G(k+1)=\mu^{n}\left(\operatorname{Rad}_{i}(k)\right) E V N(k+1) \\
& +\mu^{d}\left(\operatorname{Rad}_{i}(k)\right) E V D(k+1)
\end{aligned}
$$

donde $E V G(k+1)$ es la evapotranspiración global y

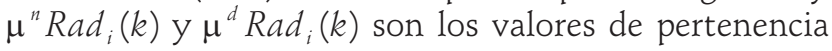
del selector difuso para los submodelos de la noche y el día, respectivamente. En este método se utilizan tres cúmulos para cada submodelo (día, noche) y el parámetro difuso $m=2.5$.
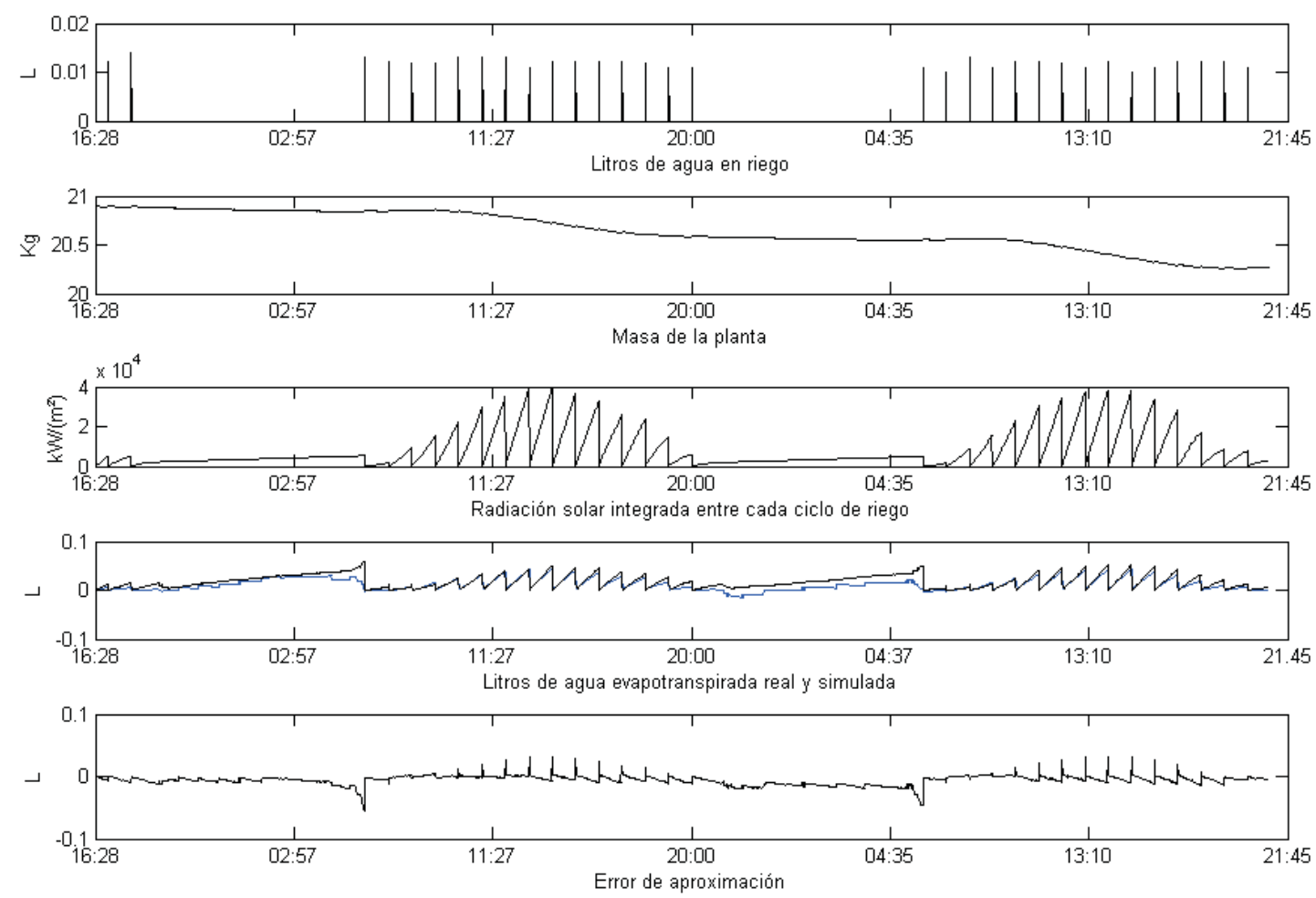

Figura 6. Evapotranspiración utilizando temperatura y humedad relativa 
DOI: http://dx.doi.org/10.22201/fi.25940732e.2010.11n2.011

Una estructura neurodifusa para modelar la evapotranspiración instantánea en invernaderos

La identificación de los parámetros antecedentes y consecuentes del submodelo que corresponde al periodo nocturno, se realizó con los datos del 15 de junio de 2005 desde las 21:52 hasta las 01:15 del 16 de junio de 2005.

Para el submodelo diurno se tomaron los datos del día 16 de junio de 2005 desde las 06:01 hasta las 13:59 del mismo día.

La validación de los submodelos obtenidos se efectuó con los datos del día 15 de junio de 2005 desde las 18:28, hasta las 20:55 del 17 de junio de 2005.

Los resultados del modelo neurodifuso de evapotranspiración instantánea se pueden ver en la figura 6 . El número de muestra $k=1$ corresponde a la muestra de las 18:28 del día 15 de junio de 2005. En la figura 6 se muestran los ciclos de irrigación con una cantidad de agua en litros ( $L$ ). Si se considera, la densidad del agua evapotranspirada igual a uno, entonces se puede aproximar la cantidad de agua como $1 \mathrm{gr}=1 \mathrm{ml}$.

Debido a que el modelo neurodifuso propuesto tiene también como objetivo proporcionar datos precisos de la salida con la mínima cantidad de variables en la entrada, en los siguientes resultados que se muestran en simulación en la figura 7 , se suplieron las variables de temperatura y humedad por la variable del Déficit de
Presión de Vapor Se utilizaron los mismos periodos de los datos de aprendizaje y de validación del modelo de evapotranspiración mostrado previamente. De esta forma, también se mejoró la varianza del error y la media del error como índices de calidad en la aproximación con el mismo periodo de experimentación. La estructura del modelo, donde se utiliza el $D P V$ es bastante simple en cuanto al número de parámetros en los antecedentes y consecuentes.

La figura 7 muestra los resultados de la simulación con esta reducción en el número de variables. La variable contiene información del microclima del invernadero, la cual es sumamente útil para relacionar y predecir la sanidad y calidad de cultivo, a nivel de plagas, hongos $y$ deformaciones en los frutos por inadecuada polinización como se ilustra en López-Morales et al. (2008).

\section{Análisis de los resultados obtenidos}

En los experimentos realizados se determinó mediante la comparación del error de aproximación, que tres cúmulos de datos es suficiente para aproximar la evapotranspiración de forma instantánea, lo que implica submodelos con tres reglas difusas T-S.
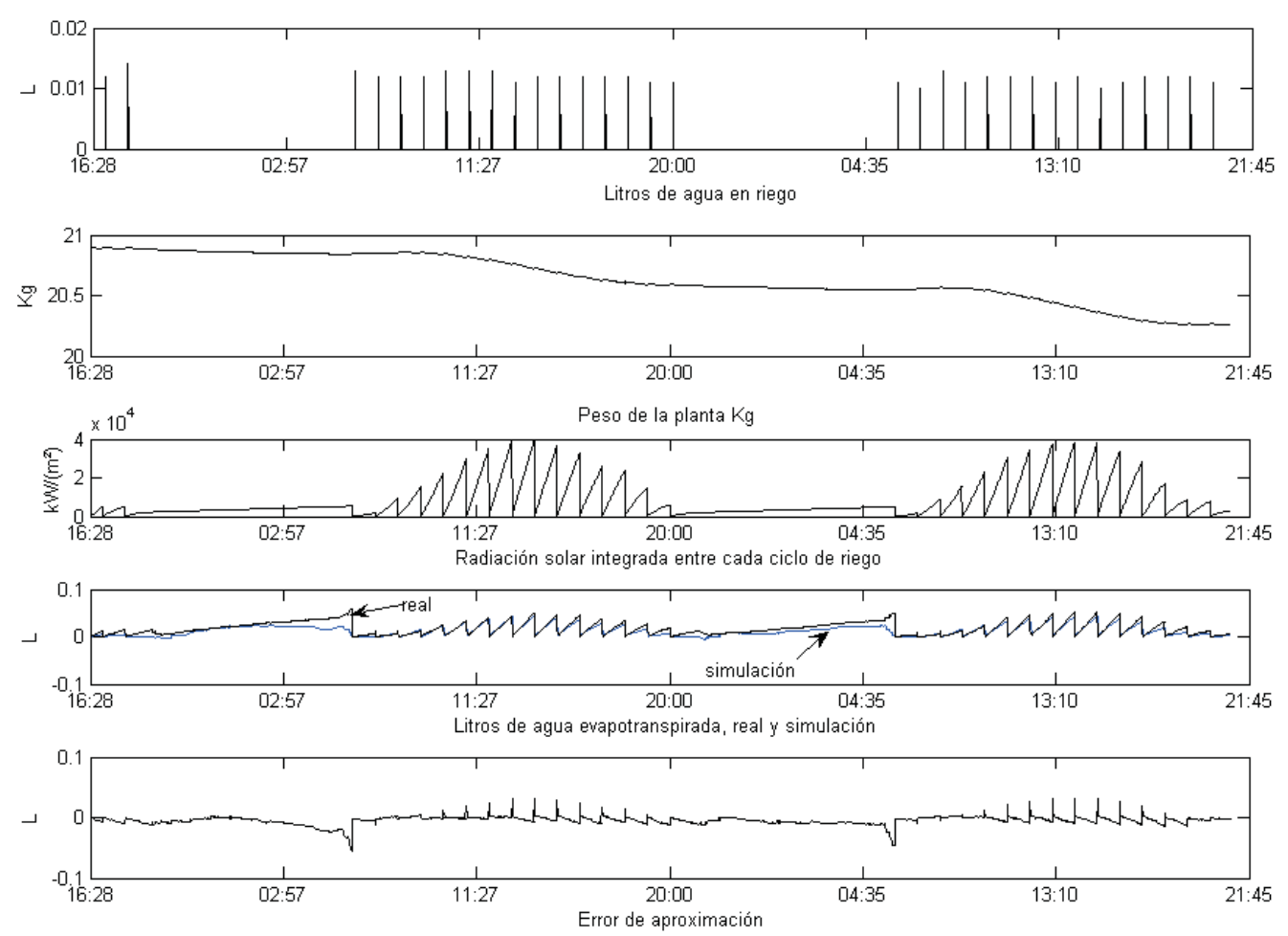

Figura 7. Evapotranspiración utilizando el DPV 
Los resultados que se muestran corresponden a dos días de experimentación, una condición inicial que se consideró es el conjunto maceta-sustrato con plena disposición de agua, es decir, después de ser saturada y drenada el agua.

La masa inicial fue de $20.898 \mathrm{~kg}$, y la masa al final de la experimentación fue de $20.262 \mathrm{~kg}$. Se realizaron $32 \mathrm{ci}-$ clos de riego (un promedio de 11.843 gr en cada manipulación de riego), con $0.3790 \mathrm{~kg}$ de agua suministrada en todo el periodo de la experimentación, donde no se obtuvo drenaje de agua.

Así que, la cantidad de agua evapotranspirada durante el periodo de manipulación se puede determinar con el siguiente balance de masa:

$$
\begin{aligned}
& M_{F}=M_{0}+M_{a d}-M_{\text {evapo }}-M_{\text {dre }} \\
& M_{\text {evapo }}=M_{0}+M_{a d}-M_{F}-M_{d r e}
\end{aligned}
$$

donde $M_{F}$ es la masa al final de la manipulación, $M_{0}$ es la masa de inicio, $M_{a d}$ es la masa adicionada durante un periodo de irrigación, $M_{\text {dre }}$ es la masa de agua drenada y $M_{\text {evapo }}$ es la masa de agua evapotranspirada. La masa total que la planta perdió en el periodo de los experimentos realizados fue de $1.016 \mathrm{~kg}$.

El déficit de agua fue grande, debido a la elevada temperatura, y en consecuencia generó un efecto importante en la evapotranspiración. La mínima temperatura y humedad relativa registrada fueron de $28{ }^{\circ} \mathrm{C}$ y $17.3 \%$, respectivamente, mientras que la máxima temperatura y humedad relativa fueron $46^{\circ} \mathrm{C}$ y $51.4 \%$, respectivamente. Estos valores están fuera de los rangos de las variables recomendadas para un cultivo de este tipo. La estimación del agua total evapotranspirada es posible obtenerla con la estructura de modelado neurodifuso presentado. El modelo toma en cuenta las variables principales que influyen en el proceso dinámico de evapotranspiración. Con ayuda de este modelo y a través del cálculo del $D P V$ es posible en una siguiente fase, regular la cantidad de agua que se suministra a una planta (y enseguida a un cultivo) mientras se conserva un rango deseable del $D P V$, para asegurar condiciones óptimas de desarrollo y sanidad del cultivo.

\section{Conclusiones y perspectivas}

La estructura neurodifusa para modelar la evapotranspiración de una sola planta (testigo) en un invernadero, como aquí se reporta, puede servir de referencia para conocer la dinámica de evapotranspiración en un cultivo que se encuentra con las mismas condiciones de la planta testigo. Sin embargo, esta dinámica tendrá algunas modificaciones al tomarse en cuenta el cultivo completo (Medrano et al., 2004) dentro del invernadero, debido a que cada planta del cultivo que es hidratada adecuadamente; en plena transpiración funciona como un pequeño regulador de temperatura. Una extensión directa del modelo que se propone es la evapotranspiración, regulando el microclima del invernadero con referencia a los perfiles sugeridos en la literatura que permiten un estado de sanidad de la producción.

Al tomar en cuenta la medición del agua drenada de la planta testigo en una balanza independiente a la medición de la masa evapotranspirada, con el fin de introducir una consigna de drenaje mínima en los ciclos de riego para minimizar la cantidad de agua drenada entre cada ciclo de riego, resultaría en una minimización de agua drenada por planta en un cultivo de dimensiones comerciales, y en consecuencia, un ahorro del agua total utilizada. El DPV puede además ser utilizado como una consigna para realizar el control en lazo cerrado del microclima, como se demuestra en este artículo, es una variable valiosa para realizar el modelado y control del microclima para poder regular la cantidad de irrigación de un cultivo.

\section{Agradecimientos}

El trabajo de investigación de Julio Cesar Ramos Fernández, ha sido apoyado por el convenio de Cooperación Científica Internacional ECOS-NORD/ANUIESSEP-CONACyT M02:M03 (Francia y México) y PROMEP- TTT. El trabajo de investigación de Virgilio López Morales es parcialmente apoyado por el proyecto de investigación ANUIES-SEP CONACyT/ECOS-NORD M02:M03.

\section{Referencias}

Abdulelah Al-Faraj, Meyer G.E., Horts G.L. A Crop Water Stress Index for Tall Fescue (Festuca Arundinacea Schreb) Irrigation Decision-Making a Fuzzy Logic Method. Computers and Electronics in Agriculture, 32:69-84. 2004.

Babel M.S., Tantau H.J., Salokhe V.M. Water Requirement of Drip Iirrigation Tomatoes Grown in Greenhouse in Tropical Environment. Agricultural Water Management, 71(3):225-242. 2005.

Babuška R. Fuzzy Modeling for Control. Kluwer Academic Publishers. 1998.

Bahat M., Invar G., Yaniv O., Shneider M. A Fuzzy Irrigation Controller System. Engineering Application, Artificial Intelligence, 1:37-145. 2000. 
DOI: http://dx.doi.org/10.22201/fi.25940732e.2010.11n2.011

Una estructura neurodifusa para modelar la evapotranspiración instantánea en invernaderos

Castañeda M.R., Ventura R.E., Peniche V.R.R., Ruiz H.G. Fuzzy Greenhouse Climate Control System Based on a Field Programmable Gate Array. Biosystems Engineering, 94(2):165-177. 2006.

Coras-Merino P.M. Evapotranspiracion y programación de riego. Universidad Autónoma de Chapingo, Departamento de Fitotecnia. 2000.

Foulloy L., Galichet S., Titli A. Commande Floue 2, Systémes Automat. Hermes Science (Eds). 2003.

Flores j., Ojeda-Bustamante W., López I., Rojano A., Salazar I. Requerimientos de riego para tomate de invernadero. Terra Latinoamericana, 25(2):127-134. 2005.

Gary C. Strategies to Control Water and Nutrient Supplies to Greenhouse Crops. A Review, Agronomie, 21:311-321. 2001.

Jou L.J., Liao C.M., Chiu C.Y. A Boolean Algorithm Suitable for Use in Temperature-Humidity Control of a Grafted Seedling Acclimatization Chamber. Computers and Electronics in Agriculture, 48:1-18. 2004.

Körner O., Challa H. Process-Based Humidity Control Regime for Greenhouse Crops. Comp. and Electr. in Agric., 39:173-192. 2003.

Lafont F., Balmat J.F. Optimized Fuzzy Control of a Greenhouse. Fuzzy Sets and Systems, 128:47-59. 2002.

Lopez-Morales V., López-Ortega O., Ramos-Fernández J.C., Muñoz L.B. JAPIEST: An Integral Intelligent System for the Diagnosis and Control of Tomatoes Diseases and Pests in Hydroponic Greenhouses. Expert Systems with Applications, 38(4):1506-1512. 2008.

Medrano E., Lorenzo P., Sánchez G.M.C., Montero J.I. Evaluation and Modelling of Greenhouse Cucumber-Crop Transpiration Under High and Low Radiation Conditions. Scientia Horticulturae, 105:163-175. 2004.

Nuez F., Rodríguez del R. A., Tello J., Cuartero J., Segura B. El cultivo del tomate. Mundi-Prensa. 2001. Pp. 139-141.

Orgaz F., Fernández M.D., Bonachela S., Gallardo M., Federes E. Evapotranspiration of Horticultural Crops in Unheated
Plastic Greenhouse. Agricultural Water Management, 72:81-96. 2004.

Poss J.A., Russell W.B., Shouse P.J., Austin R.S., Grattan S.R., Grieve C.M., Lieth J.H., Zeng L. A Volumetric Sysimeter System (VLS): An Alternative to Weighing Lysimeters for Plant-Water Relations Studies. Computers and Electronics in Agriculture, 43:55-68. 2004.

Prenger J.J., Ling P.P. Greenhouse Condensation Control, Understanding and Using Vapor Pressure Deficit (VPD). Fact Sheet (series) AX-804. Ohio State University Extension, Columbus, OH 1-4. 2001.

Roh M.Y., Lee Y.B. Control of Amount and Frequency of Irrigation According to Integrated Solar Radiation in Cucumber Substrate Culture. Proc. Int. Sym. Plant Production in Closed Ecosystems, 332-337. 1996.

Salgado P., Boaventura-Cunha J. Greenhouse Climate Hierarchical Fuzzy Modelling Control. Engineering Practice. 2004. Pp. 1-16.

Sánchez Del Castillo F., Rebolledo E.R.E. Hidroponía. Edit. Univ. Autónoma de Chapingo. 2001.

Seginer I. The Penman-Monteith Evapotranspiration Equation as an Element in Greenhouse Ventilation Design. Biosystems Engineering, 82(4):423-439. 2002.

Sigrimis N., Anastasiou A., Rerras N. Energy Saving in Greenhouse Uusing Temperature Integration: A Simulation Survey.- Computers and Electronics in Agriculture, 26:321341. 2000.

Tantau H.J., Lange D. Greenhouse Climate Control: And Approach for Integrated Pest Management. Computers and Electronics in Agriculture, 40:141-152. 2003.

Trajkovic S., Stankovic M., Todorovic B. Estimation of FAO Blaney-Criddle b Factor by RBF Network. Journal of Irrigation and Drainage Engineering, 126:268-270. 2000.

Yen J., Langari R. Fuzzy Logic Intelligence, Control and Information. Pearson Education, Inc. 1999. 
DOI: http://dx.doi.org/10.22201/fi.25940732e.2010.11n2.011

Ramos-Fernández J.C., López-Morales V., Lafont F., Enea G. y Duplaix J.

\section{Semblanza de los autores}

Julio Cesar Ramos-Fernández. Realizó sus estudios de ingeniería eléctrica en el Instituto Tecnológico de Pachuca. Fue becario del programa PROMEP para la formación de investigadores y realizó sus estudios de doctorado en co-tutela en la Universidad del Sur Toulon Var-Francia y la Universidad Autónoma del Estado de Hidalgo-México. Sus principales temas de interés en investigación incluyen modelado y control de sistemas complejos utilizando técnicas neurodifusas con aplicaciones en tiempo real a invernaderos agrícolas, actualmente es profesor de la Universidad Politécnica de Pachuca.

Virgilio López-Morales. Realizó sus estudios de doctorado en la Ecole Centrale de Nantes-Francia. Actualmente es investigador de la Universidad Autónoma del Estado de Hidalgo-México. Sus temas principales de investigación incluyen el análisis y programación de técnicas de sistemas inteligentes distribuidos, sistemas neurodifusos y control de sistemas dinámicos.

Frédéric Lafont. Recibió en 1997 el grado de doctor en ciencias de la ingeniería por la Universidad del Sur ToulonVar-Francia. Desde 1998 es investigador en el Instituto Universitario de Tecnología de la Universidad del Sur Toulon Var-Francia. Su principal línea de investigación es la identificación y el control de sistemas con múltiples entradas y múltiples salidas con técnicas neurodifusas.

Gilles Enea. Recibió en 1967 el grado de ingeniero electricista por la escuela Supérieure Electronique, Electrotéchnique, Informatique et Hydraulique de la Universidad de Toulouse Francia. Desde 1967 a 1972, fue asistente de investigación en el Laboratoire d'Automatique et d'Analyses des Systémes en la Universidad de Toulouse. Recibió el grado de doctor ingeniero y doctor de estado en ciencias por la Universidad Paul Sabatier, Toulouse en 1970 y 1975, respectivamente. Desde 1972, es profesor en la Universidad del Sur de Toulon Var-Francia, impartiendo cursos de control automático. Fue vice-presidente de esa Universidad de 1995 a 1998. Desde 2002 a 2004 fue co-director del Laboratorio de Sistemas Información y Señales de la misma Universidad.

Jean Duplaix. Estudio en la escuela Supérieure de Cachan-France de 1970 a 1975. Es investigador de la Universidad del Sur Toulon Var- Francia. Recibió el grado de doctor en 1994 en la misma Universidad. Actualmente es profesor de matemáticas aplicadas y control automático en el Instituto Universitario de Tecnología de la misma Universidad. Un área de interés en investigación es el control óptimo en el contexto del lugar de los polos. Recientes trabajos que realiza mediante soft computing en el contexto de sistemas complejos es el modelado, identificación y control con aplicaciones al microclima en invernaderos agrícolas. 